\title{
A rational method to kinetically control the rate-determining step to explore efficient electrocatalysts for the oxygen evolution reaction
}

\author{
Nam Hee Kwon ${ }^{1}$, Minho Kim², Xiaoyan Jin', Joohyun Lim', In Young Kim, Nam-Suk Lee ${ }^{3}$, Hyungjun Kim² and \\ Seong-Ju Hwang ${ }^{1}$
}

\begin{abstract}
A novel, rational, and efficient way to explore high-performance electrocatalysts was developed by controlling the reaction kinetics of the rate-determining step (RDS). Density functional theory (DFT) calculations demonstrate that the RDS for the oxygen evolution reaction driven by transition metal hydroxides/oxides, i.e., surface adsorption of $\mathrm{OH}^{-} / \mathrm{OOH}^{*}$ species, can be significantly promoted by increasing the electrophilicity of electrocatalysts via hybridization with electron-withdrawing inorganic nanosheets. As predicted by DFT calculation, the hybridization of Ni-Fe-layered double hydroxide (LDH)/Ni-Co-LDH, with $\mathrm{RuO}_{2}$ nanosheets (1.0 wt\%) leads to significant lowering of the overpotentials to $207 / 276 \mathrm{mV}$ at $10 \mathrm{~mA} \mathrm{~cm}^{-2}$, i.e., one of the smallest overpotentials for LDH-based materials, with the increase in the current density. The necessity of a very small amount of $\mathrm{RuO}_{2}$ nanosheets (1.0 wt\%) to optimize the electrocatalyst activity highlights the remarkably high efficiency of the $\mathrm{RuO}_{2}$ addition. The present study underscores the importance of kinetic control of the RDS via hybridization with electron-withdrawing species for exploring novel efficient electrocatalysts.
\end{abstract}

\section{Introduction}

Electrocatalyst materials for the oxygen evolution reaction (OER), hydrogen evolution reaction (HER), oxygen reduction reaction (ORR), and $\mathrm{CO}_{2}$ reduction reaction have attracted enormous research interest because of their crucial roles in many energy-related technologies, such as metal-air batteries, water electrolysis, fuel cells, and artificial photosynthesis ${ }^{1,2}$. These redox reactions require the simultaneous transfer of four

\footnotetext{
Correspondence: Hyungjun Kim (linus16@kaist.ac.kr) or

Seong-Ju Hwang (hwangsju@ewha.ac.kr)

${ }^{1}$ Center for Hybrid Interfacial Chemical Structure (CICS), Department of Chemistry and Nanoscience, College of Natural Sciences, Ewha Womans University, Seoul 03760, Republic of Korea

${ }^{2}$ Department of Chemistry and Graduate School of EEWS, Korea Advanced Institute of Science and Technology (KAIST), Daejeon 34141, Republic of Korea Full list of author information is available at the end of the article.

These authors contributed equally: Nam Hee Kwon, Minho Kim
}

or two electrons, which makes them very sluggish. Although there are several noble metals and their alloys showing excellent electrocatalyst activities ${ }^{3-5}$, the high cost and limited abundance of noble metal elements frustrate their practical use. To replace these precious materials, a great deal of research effort has been devoted to exploring transition metal oxide/hydroxide-based electrocatalysts with low cost and high abundance ${ }^{6,7}$. Layered transition metal hydroxides like $\mathrm{Ni}-\mathrm{Co}$-layered double hydroxide (LDH) and $\mathrm{Ni}-\mathrm{Fe}-\mathrm{LDH}$ have received prime attention because of their excellent electrocatalytic activities for diverse electrochemical reactions, including the $\mathrm{OER}^{8}$. The absence of noble metal components renders these materials promising alternatives to commercial Ir/C as OER electrocatalysts. The great flexibility in the chemical compositions of these materials provides useful opportunities to enhance their electrocatalytic 
functionalities ${ }^{9,10}$. However, the electrocatalytic performances of these transition metal hydroxides are still inferior to those of noble metal catalyst, which prompts research efforts to improve their electrocatalytic activities. Since the functionality of electrocatalysts is generally strongly dependent on the surface area, number of active sites, and electrical conductivity, many attempts have been made to optimize these characteristics ${ }^{11,12}$. In one instance, hybridization with highly conductive carbon nanostructures was quite effective in enhancing the electrocatalytic activity of transition metal hydroxide/ oxide materials by increasing the electrical conductivity ${ }^{13}$. Also, the formation of low-dimensional nanostructures with expanded surface areas, such as exfoliated twodimensional (2D) nanosheets (NSs), is quite effective in enhancing the electrocatalytic functionality ${ }^{14}$. Despite these previous attempts, a novel synthetic strategy based on the electrocatalyst reaction mechanism is still necessary to explore highly efficient electrocatalysts using economically feasible transition metal oxides/hydroxides. According to previous mechanism studies on the OER process catalyzed by oxides and oxyhydroxides, the attachment of reaction intermediate $\mathrm{OH}^{-}$and/or $\mathrm{OOH}^{*}$ species on surface active sites has a profound effect on the reaction kinetics as the rate-determining step $(\mathrm{RDS})^{15,16}$. Thus, it is crucial to tailor the binding affinities of sites on the surface of metal hydroxides for these intermediate species to improve the OER functionality. Since hydrophilic $\mathrm{RuO}_{2}$ NSs can act as an efficient electron reservoir for many inorganic solids ${ }^{17,18}$, hybridization with this electron-withdrawing NS is expected to be quite effective in enhancing the electrophilicity of transition metal hydroxides/oxides via interfacial electron transfer between hybridized NSs. The resulting increase in the electrophilicity of the electrocatalyst would be quite advantageous in promoting the binding of nucleophilic $\mathrm{OH}^{-}$and/or $\mathrm{OOH}^{*}$ species on the surface, leading to remarkable enhancement of the OER electrocatalytic activity. Thus, hybridization with this electron-withdrawing, inorganic NS can provide a novel, effective way to enhance the OER electrocatalytic activity of transition metal hydroxides/oxides. Although there are several previous reports about the synthesis of LDH-based hybridtype electrocatalysts for OERs ${ }^{10-14}$, no attempt has been made to improve the reaction kinetics of the OER process via fine-tuning the electrophilicity of the LDH. At the time of this submission, we are unaware of any other report about the enhancement of the electrocatalyst functionality and RDS kinetics using transition metal hydroxides/oxides via hybridization with inorganic NSs based on detailed speculation of the reaction mechanism.

Here we report an efficient method to explore economically feasible transition metal hydroxide/oxide-based OER electrocatalysts with unusually low overpotential via kinetic control of the RDS of the electrocatalytic reaction. The effects of hybridization with $\mathrm{RuO}_{2} \mathrm{NSs}$ on the mechanism of the OER process were quantitatively investigated with theoretical density functional theory (DFT) calculations. Based on these results, novel OER electrocatalysts, e.g., $\mathrm{LDH}-\mathrm{RuO}_{2}$ nanohybrids, were synthesized by hybridization of exfoliated LDH NSs with a small amount of $\mathrm{RuO}_{2}$ NSs $(\leq 1.5 \mathrm{wt} \%)$. The crystal structure, pore structure, and chemical bonding character of the resulting $\mathrm{LDH}-\mathrm{RuO}_{2}$ nanohybrids were systematically investigated to understand the effect of $\mathrm{RuO}_{2}$ hybridization on these properties. The OER activity of the obtained $\mathrm{LDH}-\mathrm{RuO}_{2}$ nanohybrid with a very small $\mathrm{Ru}$ content $(\sim 0.7 \mathrm{wt} \%)$ is one of the best among the reported values of LDH-based electrocatalysts, underscoring the remarkably high efficiency of hybridization with $\mathrm{RuO}_{2}$ NSs.

\section{Experimental procedures}

Theoretical simulations

The DFT calculations were performed using Vienna ab initio Simulation Package (VASP) 5.4.1 $1^{19,20}$ and project augmented wave function method ${ }^{21,22}$. The spinpolarized Perdew-Burke-Ernzerhof functional ${ }^{23}$ was chosen for the DFT calculation. For a better description of $\mathrm{Ni}-\mathrm{Co}-\mathrm{LDH}$, a Hubbard- $U$ correction $(\mathrm{DFT}+U)$ method $^{24}$ was included using $U=3.0$ for $\mathrm{Ni}$ and $U=3.5$ for $\mathrm{Co}^{25}$. The vacuum was set to be $15 \AA$ thick. The geometry optimization was completed until the total energy change between the two last ionic steps was $<1 \times 10^{-5} \mathrm{eV}^{-1}$. The optimized structures are illustrated in Figure S1 of the Supplementary Information, and the convergence processes are depicted in Figure S2 of Supplementary Information. The kinetic energy cutoff was $500 \mathrm{eV}$, and a $7 \times 6 \times 1$ Monkhorst-Pack grid was used. We used Gaussian smearing with a width of $0.2 \mathrm{eV}$ for stable convergence of the self-consistent field calculation. The Bader charge analysis was performed by using the method of Henkelman et al. ${ }^{26-28}$. The three-dimensional charge difference map was plotted by using VESTA v3.4. $0^{29}$, and the planar averaged charge difference was obtained by using VASPkit $0.3^{30}$. To estimate the Gibbs free energy change of the reduction steps, we calculated the zero-point correction energy and entropic contribution at $298.15 \mathrm{~K}(\Delta G=\Delta E+\Delta \mathrm{ZPE}-\mathrm{T} \Delta \mathrm{S}$ at standard condition). The reference potential was set as the half reaction for the reversible hydrogen electrode (RHE) under alkaline conditions $\left(2 \mathrm{H}_{2} \mathrm{O}(\mathrm{l})+2 \mathrm{e}^{-} \rightarrow \mathrm{H}_{2}(\mathrm{~g})+\right.$ $\left.2 \mathrm{OH}^{-}(\mathrm{aq})\right)$ by relating the chemical potential of the $\mathrm{OH}^{-}$ species as $\mu\left(\mathrm{OH}^{-}\right)=\mu\left(\mathrm{H}_{2} \mathrm{O}\right)-1 / 2 \mu\left(\mathrm{H}_{2}\right)+\mu\left(\mathrm{e}^{-}\right)+\mathrm{e} U$, where $U$ is the external bias voltage. The solvation energy of water was adopted from the experimental heat of vaporization ${ }^{31}$. The reference energy for the gaseous $\mathrm{O}_{2}$ was corrected to yield the calculated total energy sum of 
the OER pathway as $1.229 \mathrm{eV}$ per electron ${ }^{32}$ because of the well-known difficulties in obtaining an accurate energy of open-shell triplet $\mathrm{O}_{2}$ within $\mathrm{DFT}^{33}$.

\section{Sample preparation}

The precursors of the $\mathrm{RuO}_{2} \mathrm{NSs}, \mathrm{Ni}-\mathrm{Co}-\mathrm{LDH}$, and $\mathrm{Ni}-\mathrm{Fe}-\mathrm{LDH}$ NSs were prepared by exfoliation processes of the corresponding layered solids ${ }^{34-36}$. The exfoliation of $\mathrm{Ni}-\mathrm{Co}-\mathrm{LDH} / \mathrm{Ni}-\mathrm{Fe}-\mathrm{LDH}$ was achieved with a dispersion of the pristine LDH materials in formamide, whereas the exfoliated $\mathrm{RuO}_{2} \mathrm{NSs}$ were obtained by intercalation of tetrabutylammonium ions into protonated $\mathrm{Na}_{0.2} \mathrm{RuO}_{2}{ }^{35}$. The exfoliated LDH NSs possessed a positive layer charge, whereas the exfoliated $\mathrm{RuO}_{2}$ NSs were negatively charged (see Figure S3 in the Supplementary Information). The opposite surface charges of these NSs made their electrostatically driven self-assembly possible. As illustrated in Fig. $1, \mathrm{Ni}-\mathrm{Co}-\mathrm{LDH}-\mathrm{RuO}_{2}$ nanohybrids with variable $\mathrm{RuO}_{2}$ contents were synthesized by restacking two kinds of colloidal suspensions of oppositely charged LDHs and $\mathrm{RuO}_{2} \mathrm{NSs}$ for 1 day at room temperature. The resulting nanohybrids with $\mathrm{RuO}_{2}$ contents of $0.5,1.0$, and $1.5 \mathrm{wt} \%$ were denoted as NCR05, NCR10, and NCR15, respectively. A variable amount of $0.1 \mathrm{M} \mathrm{Na} \mathrm{Na}_{2} \mathrm{CO}_{3}$ solution was also added to maintain the charge neutrality. The obtained precipitates were washed with ethanol and distilled water and dried at $50^{\circ} \mathrm{C}$ under vacuum. Additionally, the exfoliated Ni-Fe-LDH NSs were also hybridized with $\mathrm{RuO}_{2}$ NSs. The resulting Ni-Fe-LDH- $\mathrm{RuO}_{2}$ nanohybrid with a $\mathrm{RuO}_{2}$ content of $1.0 \mathrm{wt} \%$ was denoted as NFR10.

\section{Characterization}

The zeta potentials of the colloidal suspensions of NSs were measured using a Malvern Zetasizer Nano ZS (Malvern, UK) instrument. The crystal structures of the present materials were examined by powder X-ray diffraction (XRD) analysis (Rigaku D/Max-2000/PC, Nifiltered $\mathrm{Cu} \mathrm{K} \alpha$ radiation, $25^{\circ} \mathrm{C}$ ). The crystal morphologies of the obtained materials were probed with field emissionscanning electron microscopy (FE-SEM) using a JEOL

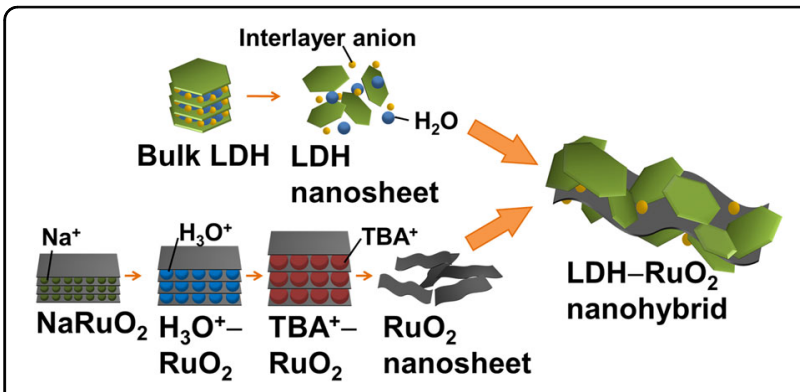

Fig. 1 Experimental schematic diagram. Schematic diagram of the exfoliation-restacking route to $\mathrm{LDH}-\mathrm{RuO}_{2}$ nanohybrids
JSM-6700F microscope. High-resolution transmission electron microscopy (HR-TEM) images of the present materials were obtained with a JEOL JEM-2100F microscope at an accelerating voltage of $200 \mathrm{kV}$. Energy dispersive spectrometry (EDS)-line scanning and energyfiltered transmission electron microscopy (EFTEM)elemental mapping analyses were carried out to probe the spatial elemental distributions of the hybrid materials. The chemical bonding state of the $\mathrm{RuO}_{2} \mathrm{NSs}$ in the present nanohybrids was investigated by Fourier transformed-infrared spectroscopy using a JASCO FT/IR6100 FT spectrometer. The local structures of the nanohybrids were studied using X-ray absorption spectroscopy (XAS). All the XAS data were collected at beam line 10C of the Pohang Accelerator Laboratory (Pohang, Korea) in transmission mode. The surface areas of the present materials were monitored using $\mathrm{N}_{2}$ adsorption-desorption isotherm measurements at $77 \mathrm{~K}$ (Micromeritics ASAP 2020). The interfacial charge transfer between the LDHs and $\mathrm{RuO}_{2} \mathrm{NSs}$ was examined with X-ray photoelectron spectroscopy (XPS, Thermo VG, UK).

\section{Measurement of the electrocatalytic activity}

The electrochemical measurements were performed at room temperature using an IVIUM analyzer with a typical three-electrode cell. A rotating ring-disk electrode-3A (ALS) was used as a rotator. The catalyst ink was prepared by dispersing $7 \mathrm{mg}$ of active material, $3 \mathrm{mg}$ of carbon black (Vulcan-XC72R), and $25 \mu \mathrm{l}$ of a $5 \mathrm{wt} \%$ Nafion solution (Sigma-Aldrich) in an isopropanol/water (1/4, $\mathrm{vol} / \mathrm{vol}$ ) mixed solvent by sonication for $1 \mathrm{~h}$. A total of $10 \mu \mathrm{l}$ of catalyst ink was dropped onto a glassy carbon (GC) electrode (3 mm, ALS), which was oven-dried at $50{ }^{\circ} \mathrm{C}$. A Pt wire and saturated calomel electrode were used as the counter electrode and reference electrode, respectively. All measurements were carried out in an $\mathrm{O}_{2}$ saturated $1 \mathrm{M} \mathrm{KOH}$ solution. The potentials were referenced to the RHE according to the Nernst equation, $E$ $(\mathrm{RHE})=E(\mathrm{SHE})+0.241_{2}+0.059 \times \mathrm{pH} \quad\left(\right.$ at $\left.25^{\circ} \mathrm{C}\right)$. The electrocatalytic OER performance was examined by linear sweep voltammetry (LSV), which was measured with a rotating speed of $1600 \mathrm{rpm}$ at a scan rate of $5 \mathrm{mV} \mathrm{s}^{-1}$ from 1.15 to $1.75 \mathrm{~V}$ (vs. RHE) for NCR and from 1.15 to $1.65 \mathrm{~V}$ (vs. RHE) for NFR. The current density $(j)$ was calculated from the ratio of the current over the surface area of the GC $\left(0.071 \mathrm{~cm}^{2}\right)$. The dependence of the charging current density on the scan rate was measured by cyclic voltammetry (CV) in the potential range from 0.9964 to $1.0964 \mathrm{~V}$ (vs. RHE) to cover the open circuit potential of $1.0744 \mathrm{~V}$ (vs. RHE). Electrochemical impedance spectroscopy (EIS) data were collected at $1.6 \mathrm{~V}$ (vs. RHE) for NCR and $1.5 \mathrm{~V}$ (vs. RHE) for NFR with a frequency range of $0.1-10000 \mathrm{~Hz}$. Chronopotentiometry was performed at $10 \mathrm{~mA} \mathrm{~cm}^{-2}$ using rotating electrodes with $\mathrm{O}_{2}$ bubbling. 


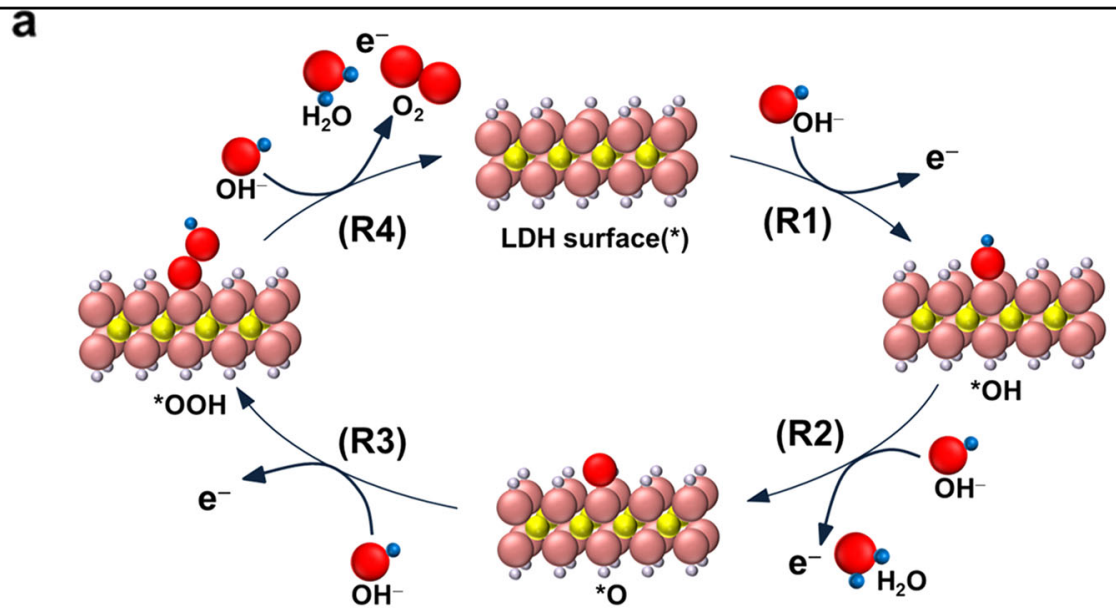

b

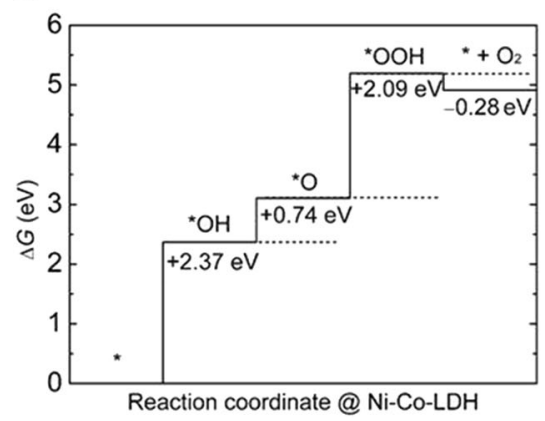

C

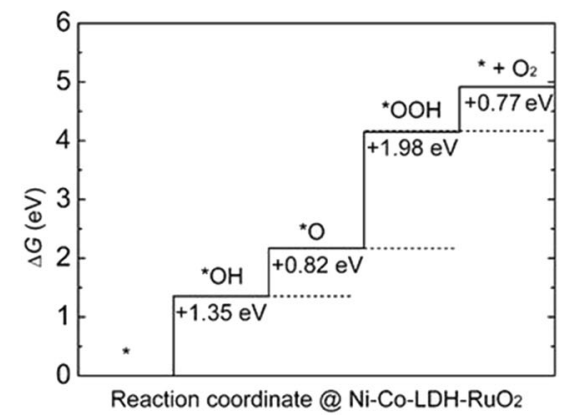

Fig. 2 DFT calculated reaction energies of the OER with corresponding optimized structures. a The overall OER mechanism on the surface of $\mathrm{LDH}$ in alkaline media and reaction energy diagram of the OER. Energy profiles of each reaction step on $\mathbf{b}$ the $\mathrm{Ni}-\mathrm{Co}-\mathrm{LDH}$ monolayer slab and $\mathbf{c ~ N i - C o - L D H - R u O} 2$ nanohybrid

\section{Results and discussion DFT calculations}

The effect of hybridization with $\mathrm{RuO}_{2}$ NSs on the operation mechanism of the OER catalytic activity of $\mathrm{Ni}-\mathrm{Co}-\mathrm{LDH}$ was theoretically investigated by DFT calculations. According to a previous study on the OER pathway in alkaline media ${ }^{37,38}$, the OER pathway is described as the adsorption of successive intermediate species on the catalyst (*) as follows (see also Fig. 2a);

$$
\begin{aligned}
& *+\mathrm{OH}^{-} \rightarrow * \mathrm{OH}+\mathrm{e}^{-} \\
& * \mathrm{OH}+\mathrm{OH}^{-} \rightarrow * \mathrm{O}+\mathrm{H}_{2} \mathrm{O}+\mathrm{e}^{-} \\
& * \mathrm{O}+\mathrm{OH}^{-} \rightarrow * \mathrm{OOH}+\mathrm{e}^{-} \\
& * \mathrm{OOH}+\mathrm{OH}^{-} \rightarrow *+\mathrm{O}_{2}+\mathrm{H}_{2} \mathrm{O}+\mathrm{e}^{-}
\end{aligned}
$$

We consider the catalytically active site as the deprotonated oxygen atom on the LDH surface ${ }^{37}$. As shown in Fig. 2b, the first step of the OER pathway on the Ni-CoLDH monolayer, i.e., (R1), is calculated to be the most energetically unfavorable step, providing a threshold energy of $2.37 \mathrm{eV}$. Hybridization with $\mathrm{RuO}_{2}$ makes the third step (R3) the most energetically unfavorable step with a threshold energy of $1.98 \mathrm{eV}$ (Fig. 2c). As expected from the previous study ${ }^{17}$, this result indicates an increase in the binding energy of $\mathrm{OH}^{-}$species on the $\mathrm{LDH}$ layer upon hybridization with $\mathrm{RuO}_{2} \mathrm{NSs}$. Indeed, the distance between the oxygen atoms of the $\mathrm{OH}^{-}$species and the $\mathrm{Ni}-\mathrm{Co}-\mathrm{LDH}$ surface decreases from 1.499 to $1.476 \AA$ after the hybridization of $\mathrm{LDH}$ with the $\mathrm{RuO}_{2}$ slab, as shown in Figures S1a and S1b in the Supplementary Information. The origin of the enhanced binding energy of $\mathrm{OH}^{-}$species on the nanohybrids can be found in the electronic interaction between the $\mathrm{LDH}$ and $\mathrm{RuO}_{2}$ layers. From the DFT-optimized structures, we find that the interlayer distance between $\mathrm{RuO}_{2}$ and LDH is close enough to allow direct orbital-orbital interaction; the top-most oxygen atoms of $\mathrm{RuO}_{2} \mathrm{NSs}$ and bottom-most hydrogen atoms of $\mathrm{Ni}-\mathrm{Co}-\mathrm{LDH}$ NSs are separated by only $1.86 \AA$.

As shown in the density of state analysis of Fig. 3, the occupied Ni $3 d$ states in the middle of the bandgap of $\mathrm{Ni}$-Co-LDH (Fig. 3a) are located close to the lowest unoccupied states of the $\mathrm{RuO}_{2}$ slab (Fig. 3b). Thus, after hybridization, significant mixing of the electronic structures of $\mathrm{RuO}_{2}$ and LDH occurs (Fig. 3c), resulting in charge transfer from LDH to $\mathrm{RuO}_{2}$ (Fig. 3d) and a formation of states at the Fermi level elevating the electrical conductivity of the catalyst. To quantify the amount of 
a

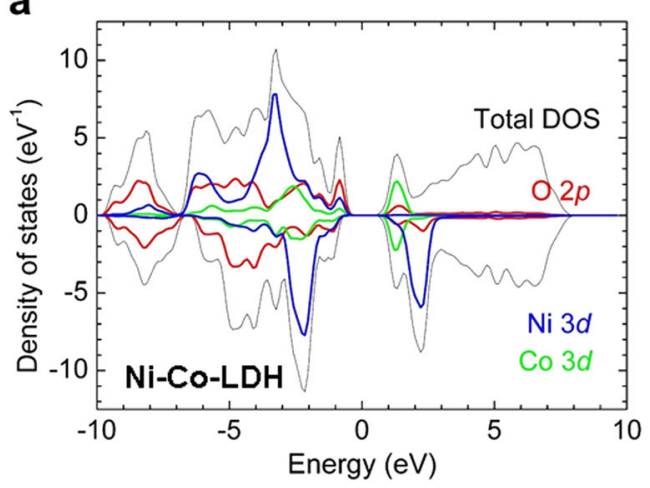

b

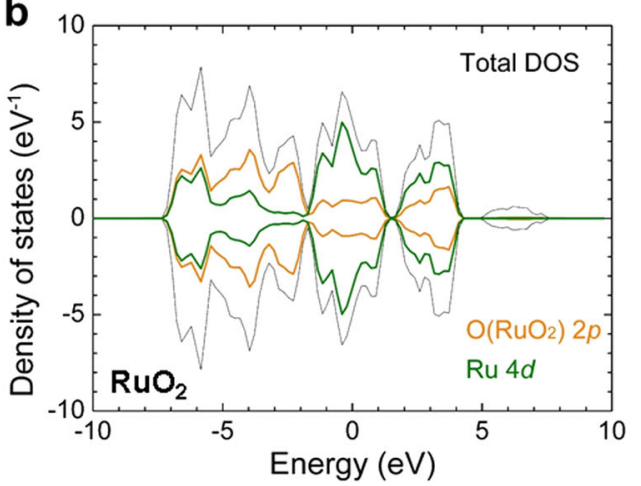

C

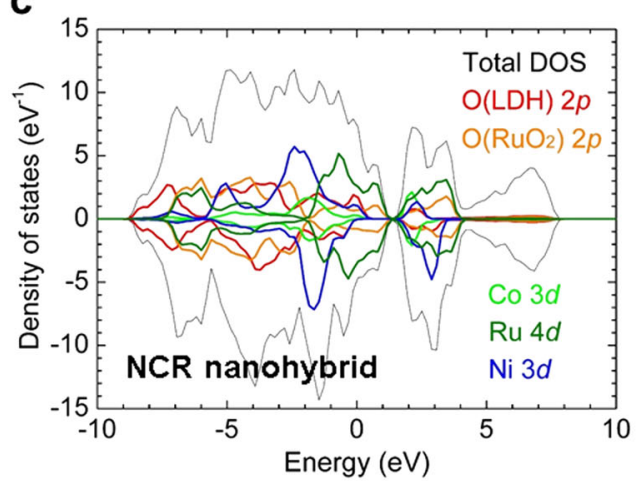

d

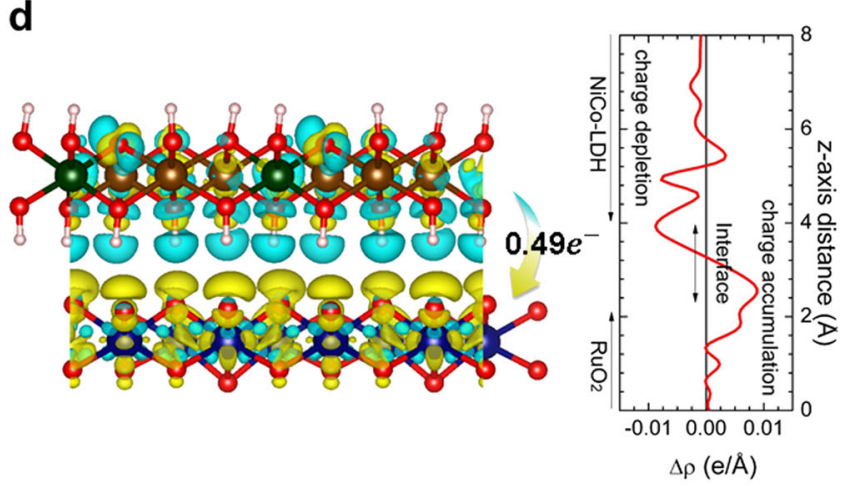

Fig. 3 The density of states (DOS) and charge difference map of the NCR nanohybrid model. The DOS of the $\mathbf{a} \mathrm{Ni}-\mathrm{CO}-\mathrm{LDH}$ slab model, $\mathbf{b} \mathrm{RuO} 2$ NS model, and $\mathbf{c}$ NCR nanohybrid model, where the Fermi level is set to zero. $\mathbf{d}$ The three-dimensional charge difference map at the isosurface level of $1.00 \Delta \rho_{\max }(100 \%$ of the maximum value of $\Delta \rho)$, where charge accumulation and depletion are shown in yellow and cyan, respectively, and the corresponding planar averaged charge difference along the surface normal direction is shown on the right. Atoms in the structure are colored by white for $\mathrm{H}$, red for $\mathrm{O}$, brown for $\mathrm{Ni}$, green for $\mathrm{Co}$, and navy for $\mathrm{Ru}$

charge transfer upon hybridization, we further performed a Bader charge analysis for the NCR nanohybrid, showing that $0.49 \mathrm{e}^{-}$is transferred to the $\mathrm{RuO}_{2} \mathrm{NSs}$ from Ni-Co$\mathrm{LDH}$. It was also found that the deprotonated oxygen atom (which serves as the catalytically active center of $\mathrm{Ni}-\mathrm{Co}-\mathrm{LDH})$ loses the most charge $\left(0.08 \mathrm{e}^{-}\right)$among the oxygen atoms of $\mathrm{Ni}-\mathrm{Co}-\mathrm{LDH}$. Thus, the present DFT calculation confirmed our speculation that charge transfer from $\mathrm{Ni}-\mathrm{Co}-\mathrm{LDH}$ to $\mathrm{RuO}_{2} \mathrm{NSs}$ makes the oxygen atom of $\mathrm{LDH}$ more electrophilic, and thus, hybridization with $\mathrm{RuO}_{2} \mathrm{NSs}$ enhances the adsorption of $\mathrm{OH}^{-}$on the $\mathrm{Ni}-\mathrm{Co}-\mathrm{LDH}$, leading to a decrease in the overpotential for OER.

\section{Structural and morphological characterizations of the NCR nanohybrids}

Based on the results of the DFT calculations, the exfoliated Ni-Co-LDH NSs were hybridized with a small amount of $\mathrm{RuO}_{2} \mathrm{NSs}$ to experimentally verify the usefulness of $\mathrm{RuO}_{2}$ NS hybridization in improving the OER efficiency of a LDH material. As plotted in Fig. 4a, all the present NCR nanohybrids as well as the pristine
$\mathrm{Ni}-\mathrm{Co}-\mathrm{LDH}$ show XRD peaks typical of the Ni-Co-LDH phase, indicating stabilization of the $\mathrm{LDH}$ in the present nanohybrids. In contrast to the Bragg reflections of the LDH phase, the XRD peaks of layered $\mathrm{RuO}_{2}$ are not discernible, reflecting the homogeneous dispersion of $\mathrm{RuO}_{2}$ NSs and/or its low concentration below the detection limit. However, the incorporation of $\mathrm{RuO}_{2} \mathrm{NSs}$ into the present NCR nanohybrids is obviously evidenced by the $\mathrm{Ru}$ K-edge extended X-ray absorption fine structure and EDS-line scanning analyses, which show the presence of $\mathrm{RuO}_{2} \mathrm{NSs}$ in these materials (see Figures S4 and S5 in the Supplementary Information). As shown in the FE-SEM images of Fig. $4 \mathrm{~b}$, the exfoliation of $\mathrm{Ni}-\mathrm{Co}-\mathrm{LDH}$ into monolayered NSs induces a marked decrease in the lateral dimension of the LDH crystallites due to severe elastic deformation caused by the expansion of the interlayer spacing during the exfoliation process (see Figure S6 in the Supplementary Information). The NCR nanohybrids show house-of-cards-type stacking structures, reflecting the creation of mesopores upon hybridization. The HRTEM image clearly demonstrates the intimate stacking of the oppositely charged $\mathrm{Ni}-\mathrm{Co}-\mathrm{LDH}$ and $\mathrm{RuO}_{2} \mathrm{NSs}$. 


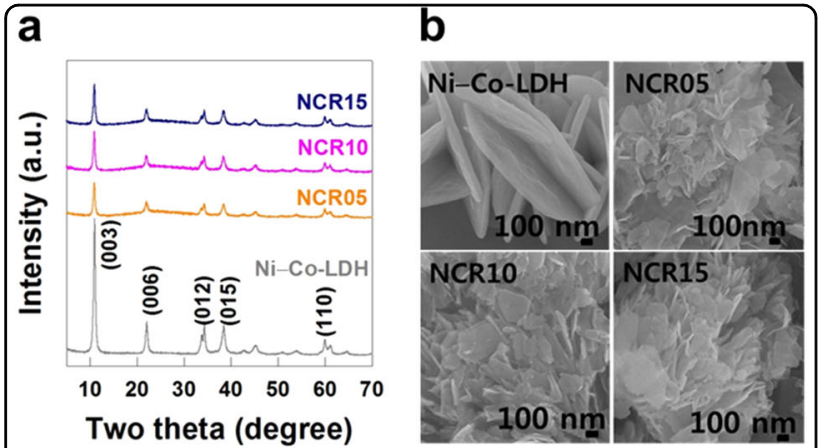

C

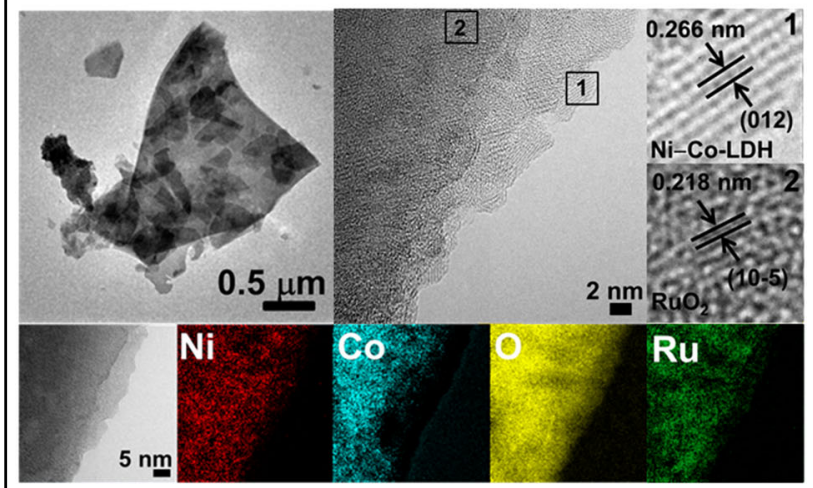

d

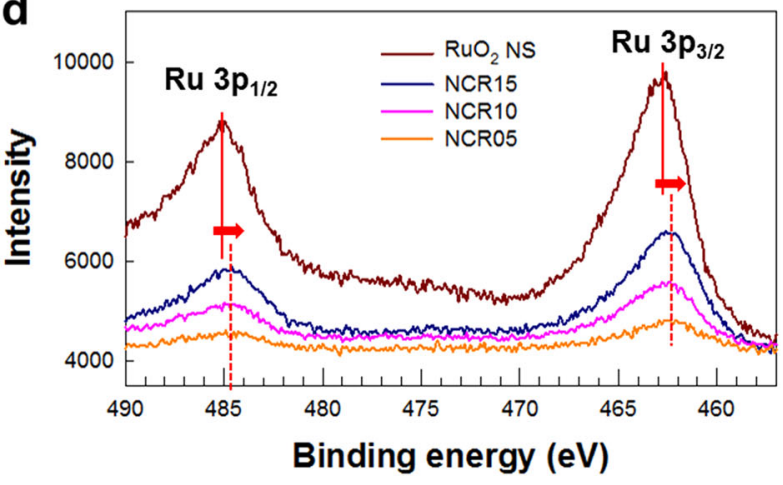

Fig. 4 Hybridization of cationic Ni-Co-LDH with anionic $\mathrm{RuO}_{2} \mathrm{NS}$. a Powder XRD patterns of NCR nanohybrids and the pristine Ni-CoLDH. $\mathbf{b}$ FE-SEM images of NCR nanohybrids and the pristine Ni-CoLDH. c HR-TEM images and EFTEM-elemental maps of NCR10, and d Ru $3 p$ XPS spectra of $\mathrm{RuO}_{2}$ NSs and NCR nanohybrids

As seen clearly from Figure S7 in the Supplementary Information, the exfoliated $\mathrm{RuO}_{2}$ NSs have a large lateral dimension of several micrometers, which is much greater than that of the Ni-Co-LDH NSs ( 100-200 nm). As illustrated in Fig. 4c, clear lattice fringes corresponding to the $(0,1,2)$ plane of $\mathrm{Ni}-\mathrm{Co}-\mathrm{LDH}$ and the $(1,0,-5)$ plane of $\mathrm{RuO}_{2}$ are obviously observed in the present HRTEM image, confirming the intimate hybridization between the two kinds of NSs. Moreover, the EFTEMelemental mapping analysis provides further evidence for nanoscale mixing between the $\mathrm{LDH}$ and $\mathrm{RuO}_{2}$ NSs, showing the uniform distribution of $\mathrm{Ru}, \mathrm{Ni}, \mathrm{Co}$, and $\mathrm{O}$ in all parts of NCR10. The molar ratios of $\mathrm{LDH}: \mathrm{RuO}_{2}$ were determined to be 1:0.007, 1:0.013, and 1:0.021 for NCR05, NCR10, and NCR15, respectively.

The stabilization of Ni-Co-LDH in the NCR nanohybrid was further evidenced by the observation of the typical IR band of this LDH phase (Figure S8 in the Supplementary Information). Of prime importance is that the weak but distinct IR band of the $\mathrm{RuO}_{2}$ phase appears at $470 \mathrm{~cm}^{-1}$ for both NCR10 and the $\mathrm{RuO}_{2} \mathrm{NSs}$, confirming the incorporation of $\mathrm{RuO}_{2} \mathrm{NSs}$ into the present nanohybrid. The coexistence of Ni-Co-LDH and $\mathrm{RuO}_{2}$ NSs in the present NCR nanohybrids was also confirmed by the $\mathrm{Ni} \mathrm{K}$-, Co K-, and $\mathrm{Ru} \mathrm{K}$-edge X-ray absorption near-edge structure (XANES) spectroscopic analyses (see Figure S9 in the Supplementary Information). In the Ru K-edge XANES spectra, all the NCR nanohybrids exhibit spectral features similar to those of the layered $\mathrm{Na}_{0.2} \mathrm{RuO}_{2}$, indicating the maintenance of the original layered lattice structure of the $\mathrm{RuO}_{2} \mathrm{NSs}$ upon hybridization with LDH. Similarly, in the Ni K- and Co K-edge XANES region, the overall spectral features of the NCR nanohybrids are nearly identical to those of the pristine $\mathrm{Ni}-\mathrm{Co}-\mathrm{LDH}$, underscoring the incorporation of the $\mathrm{Ni}-\mathrm{Co}-\mathrm{LDH}$ lattice in the present nanohybrids. The $\mathrm{N}_{2}$ adsorption-desorption isotherm measurements demonstrate that all the present NCR nanohybrids possess slightly larger Brunauer-Emmett-Teller surface areas $\left(\sim 35 \mathrm{~m}^{2} \mathrm{~g}^{-1}\right)$ than that of the pristine $\mathrm{Ni}-\mathrm{Co}-\mathrm{LDH}$ $\left(\sim 30 \mathrm{~m}^{2} \mathrm{~g}^{-1}\right)$, Figure S10 in the Supplementary Information. Despite the very small amount of $\mathrm{RuO}_{2}$ NSs incorporated, hybridization with $\mathrm{RuO}_{2}$ NSs leads to a slight but distinct increase in the surface area, indicating the usefulness of $\mathrm{RuO}_{2}$ addition in enhancing the porosity of $\mathrm{LDH}$ materials.

The interfacial charge transfer between the hybridized components was experimentally verified with a surfacesensitive XPS technique. As plotted in Fig. 4d, two $\mathrm{Ru} 3 p_{3 / 2}$ and $3 p_{1 / 2}$ XPS peaks show lower binding energies of 462.4 and $484.8 \mathrm{eV}$ for NCR05, 462.5 and $484.8 \mathrm{eV}$ for NCR10, and 462.4 and $484.7 \mathrm{eV}$ for NCR15 compared with those for the $\mathrm{RuO}_{2}$ NSs (462.7 and $485.1 \mathrm{eV}$ ), highlighting the $\mathrm{Ru}$ oxidation state is lowered upon hybridization with Ni-Co-LDH NS. Conversely, the Ni $2 p$ and Co $2 p$ XPS peaks display a slight blueshift upon hybridization, confirming an electron transfer from Ni-Co-LDH to $\mathrm{RuO}_{2} \mathrm{NSs}$, Figure S11 in the Supplementary Information. To further confirm the minute spectral changes upon hybridization with a small amount of $\mathrm{RuO}_{2}$ NSs (1 wt\%), the reference nanohybrid of $\mathrm{Ni}-\mathrm{Co}-\mathrm{LDH}$ with a much larger amount of $\mathrm{RuO}_{2} \mathrm{NSs}(10 \mathrm{wt} \%)$ was prepared and studied via Ni $2 p$ and Co $2 p$ XPS measurements. As shown in Figure S11 in the Supplementary Information, 
a

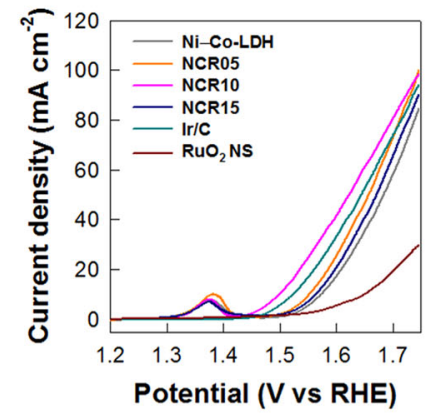

d

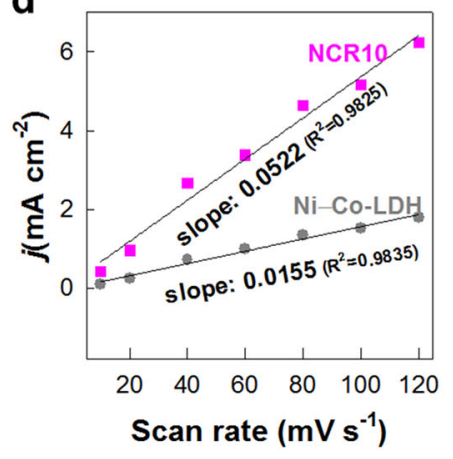

b
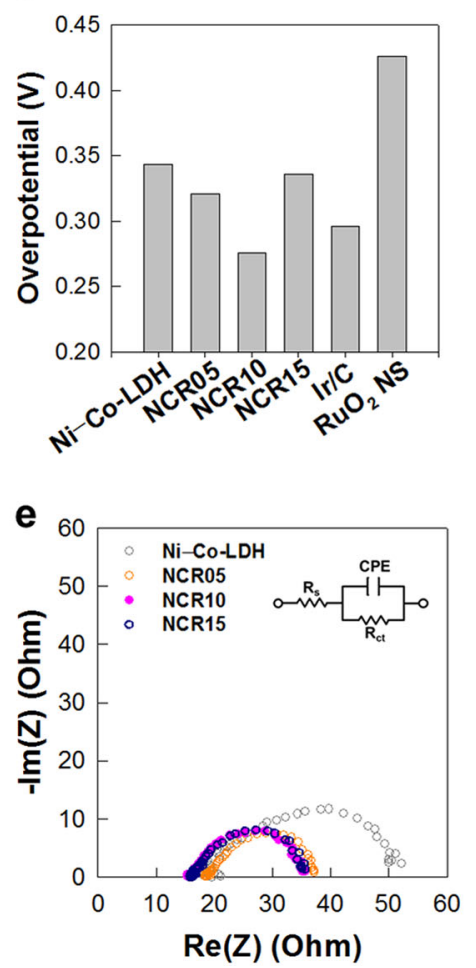

c

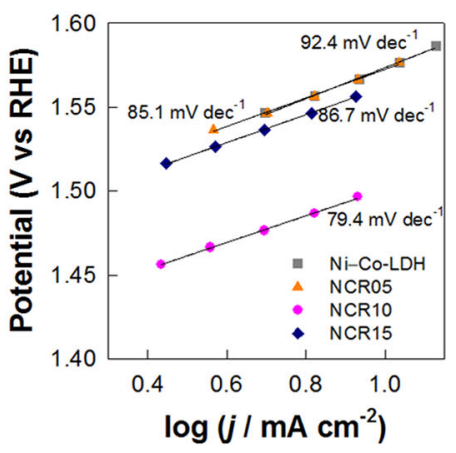

$\mathbf{f}$

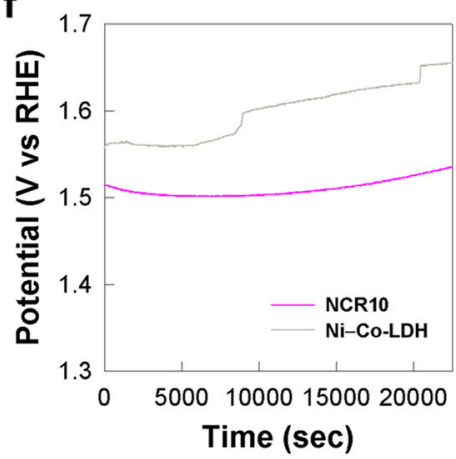

Fig. 5 Electrocatalytic properties of the NCR nanohybrids. a LSV curves. b Overpotential required to reach $j=10 \mathrm{~mA} \mathrm{~cm}^{-2}$. c Tafel plots of NCR nanohybrids and Ni-Co-LDH. d Charging current density differences plotted against scan rates of NCR10 and Ni-Co-LDH. e EIS spectra of NCR nanohybrids, and $\mathbf{f}$ chronopotentiometry measurements at $j=10 \mathrm{~mA} \mathrm{~cm}{ }^{-2}$ of $\mathbf{N C R} 10$ and Ni-Co-LDH

this reference material displays a much more prominent blueshift in the Ni $2 p$ and Co $2 p$ XPS peaks, confirming the increase in the oxidation states of $\mathrm{Ni}$ and $\mathrm{Co}$ upon hybridization with $\mathrm{RuO}_{2}$ NSs. The present XPS results are in good agreement with the DFT calculation results, showing interfacial electron transfer from $\mathrm{Ni}-\mathrm{Co}-\mathrm{LDH}$ to $\mathrm{RuO}_{2} \mathrm{NSs}$ (Fig. 3d).

\section{Electrochemical performance of the NCR nanohybrids}

The influence of the hybridization with $\mathrm{RuO}_{2} \mathrm{NSs}$ on the electrocatalytic activity of $\mathrm{Ni}-\mathrm{Co}-\mathrm{LDH}$ was examined for the OER process $\left(4 \mathrm{OH}^{-} \rightarrow 2 \mathrm{H}_{2} \mathrm{O}+4 \mathrm{e}^{-}+\mathrm{O}_{2} \uparrow\right)$. As plotted in Fig. 5a, all the present materials show a small peak between 1.3 and $1.4 \mathrm{~V}$ in the LSV curves, which corresponds to the oxidation of $\mathrm{Ni}^{2+}$ and $\mathrm{Co}^{3+}$ ions, confirming the main role of $\mathrm{Ni}-\mathrm{Co}-\mathrm{LDH}$ in the electrochemical activities of these materials ${ }^{39}$. The NCR nanohybrids display much smaller on-set potentials with a greater current density than that of the pristine $\mathrm{Ni}-\mathrm{Co}-$ LDH, highlighting the beneficial effect of hybridization with $\mathrm{RuO}_{2}$ NSs. Figure 5b presents the overpotentials of the present materials at a current density $(j)$ of $10 \mathrm{~mA}$ $\mathrm{cm}^{-2}$, which were taken from the LSV curves in Fig. $5 \mathrm{a}$. The overpotential of $\mathrm{Ni}-\mathrm{Co}-\mathrm{LDH}$ is lowered by the incorporation of $\mathrm{RuO}_{2} \mathrm{NSs}$. Among the present nanohybrids, NCR10 shows the best electrocatalytic performance with an overpotential of $276 \mathrm{mV}$ at $10 \mathrm{~mA} \mathrm{~cm}^{-2}$, which is $67 \mathrm{mV}$ lower than that of $\mathrm{Ni}-\mathrm{Co}-\mathrm{LDH}$, as predicted by the DFT calculations. Of prime importance is that the OER electrocatalyst performance of this material is the best with the smallest overpotential among all reported data on Ni-Co-LDH-based materials (see Table S1 in the Supplementary Information). Although $\mathrm{RuO}_{2}$ is one of the best electrocatalysts for $\mathrm{OER}^{40-42}$, the present NCR nanohybrid displays a better electrocatalytic performance for OER than the $\mathrm{RuO}_{2}$ NSs. In comparison with rutiletype $\mathrm{RuO}_{2}$ nanoparticles ${ }^{42}$, the layered $\mathrm{RuO}_{2} \mathrm{NSs}$ show a lower OER activity. Generally, there are many factors affecting the OER activity of inorganic solids, such as the crystal structure, electronic structure, particle size, and degree of hydration ${ }^{43,44}$. An excellent OER electrocatalytic activity has been reported only for rutile $\mathrm{RuO}_{2}$ nanoparticles and not for layered $\mathrm{RuO}_{2} \mathrm{NSs}^{45}$. Thus, the inferior OER activity of $\mathrm{RuO}_{2} \mathrm{NSs}$ is attributable to its unique layered structure. Considering the inferior OER activity of layered $\mathrm{RuO}_{2} \mathrm{NSs}$, the observed excellent OER electrocatalytic performance of NCR is interpreted to be a result of the synergistic coupling effect of two kinds of 
NSs. The beneficial effect of nanoscale hybridization with $\mathrm{RuO}_{2}$ NSs on the electrocatalytic activity of LDH is further evidenced by the OER electrocatalytic performance of NCR that is superior to that of a physical mixture of $\mathrm{Ni}-\mathrm{Co}-\mathrm{LDH}$ NSs and $\mathrm{RuO}_{2}$ NSs. As shown in Figure S12 in the Supplementary Information, the electrocatalytic performance of the obtained physical mixture is markedly poorer than that of the NCR nanohybrid, underscoring the importance of nanoscale mixing between $\mathrm{Ni}-\mathrm{Co}-\mathrm{LDH}$ and $\mathrm{RuO}_{2}$.

The OER catalytic kinetics of the present nanohybrids was also examined by calculating the Tafel slope. As shown in Fig. 5c, all the NCR05, NCR10, and NCR15 nanohybrids exhibit smaller Tafel values, i.e., 85.1, 79.4, and $86.7 \mathrm{mV} \mathrm{dec}^{-1}$, respectively, than the pristine $\mathrm{Ni}-\mathrm{Co}-$ $\mathrm{LDH}\left(92.4 \mathrm{mV} \mathrm{dec}^{-1}\right)$ and the physical mixture of Ni-CoLDH NSs and $\mathrm{RuO}_{2}$ NSs $\left(93.0 \mathrm{mV} \mathrm{dec}{ }^{-1}\right)$, highlighting the significant improvement in the OER kinetics upon nanoscale hybridization with $\mathrm{RuO}_{2}$ NSs. The smallest Tafel value occurs for NCR10, which is in good agreement with the high OER activity of this material. The electrochemically active surface area (ECSA) was also estimated from the plot of the charging current versus scan rate measured by $\mathrm{CV}$, and the slope of this plot is equivalent to twice that of the ECSA. As presented in Fig. 5d, the ECSA of NCR10 is $162 \%$ greater than that of $\mathrm{Ni}-\mathrm{Co}-\mathrm{LDH}$, strongly suggesting effective exposure of the edge sites of NSs upon the exfoliation-restacking process. Figure 5e shows the Nyquist plots of the NCR nanohybrids and the pristine $\mathrm{Ni}-\mathrm{Co}-\mathrm{LDH}$ at an applied potential of $1.6 \mathrm{~V}$ (vs. RHE) with the simulated fits. As shown in the EIS data in Fig. 5e, the semicircle reflecting charge transfer resistance $\left(R_{\mathrm{ct}}\right)$ decreases upon hybridization with $\mathrm{RuO}_{2}$ NSs. According to the fitting analysis using the equivalent circuit, the nanohybrids of NCR05, NCR10, and NCR15 have $R_{\mathrm{ct}}$ values of 20.6, 19.0, and $19.3 \Omega$, respectively, which are smaller than that of the pristine $\mathrm{Ni}-\mathrm{Co}-\mathrm{LDH}(34.38 \Omega)$, indicating improvement in the charge transfer kinetics at the interfacial region upon hybridization with $\mathrm{RuO}_{2} \mathrm{NSs}$. Among the present materials, NCR10 shows the smallest semicircle with the fastest electron transfer rate and smallest $R_{\mathrm{ct}}$. Since the $R_{\mathrm{ct}}$ value corresponds to the electrochemical impedance for ion migration into the interfacial region near electrochemically active sites of electrocatalysts during the OER process, this parameter is dependent on both the diffusion rate of the reactant ions into the interfacial region and the electrical conductivity of the electrocatalyst. The content of electrocatalytically active LDH NSs has a significant influence on the $R_{\mathrm{ct}}$ because promotion of the OER process will increase the ion diffusion rate into the interfacial region of the electrochemically active sites by enhancing the consumption of reactant ions. Additionally, the content of highly conductive $\mathrm{RuO}_{2} \mathrm{NSs}$ also affects the $R_{\mathrm{ct}}$ value by increasing the electrical conductivity of the NCR electrocatalyst. Thus, there must be an optimal ratio of $\mathrm{LDH} / \mathrm{RuO}_{2}$ to minimize the charge transfer resistance of NCR. The present EIS results indicate that the NCR10 nanohybrid possesses an optimal ratio of $\mathrm{RuO}_{2} / \mathrm{LDH}$ to minimize the $R_{\mathrm{ct}}$.

As plotted in the chronopotentiometry data in Fig. 5f, $\mathrm{Ni}-\mathrm{Co}-\mathrm{LDH}$ exhibits a marked increase in potential after $\sim 8000 \mathrm{~s}$, whereas the potential of NCR10 retains its initial value up to $\sim 16,500 \mathrm{~s}$, highlighting the remarkable improvement in the electrochemical stability upon hybridization with conductive $\mathrm{RuO}_{2} \mathrm{NSs}$. Over a longer period, the NCR10 material still shows good long-term stability superior to that of the pristine $\mathrm{Ni}-\mathrm{Co}-\mathrm{LDH}$, Figure S13 in the Supplementary Information. Since the LDH has poor electrical conductivity, the accumulation of charge upon the application of an electrical potential leads to redox-induced degradation of the LDH lattice and less electrochemical stability. Hybridization with highly conductive $\mathrm{RuO}_{2}$ NSs increases the electrical conductivity of the hybridized material, resulting in a decrease in the charging effect and an improvement in the electrochemical stability upon hybridization ${ }^{35}$. In addition, the decreased aggregation of LDH crystals and formation of a porous stacking structure upon hybridization with $\mathrm{RuO}_{2}$ NSs can also contribute to the enhanced electrochemical stability of the NCR nanohybrids $^{46,47}$. Of prime importance is that NCR10 displays a higher stability than rutile $\mathrm{RuO}_{2}$ and commercial $\mathrm{Ir} / \mathrm{C}$ electrocatalysts, as shown in Figure S14 in the Supplementary Information. The present findings clearly demonstrate the high efficiency of $\mathrm{RuO}_{2} \mathrm{NSs}$ as an additive to optimize the electrocatalytic performance of LDH materials.

\section{Hybridization of cationic Ni-Fe-LDH NSs with anionic $\mathrm{RuO}_{2}$ NSs}

To verify the universal validity of hybridization with $\mathrm{RuO}_{2} \mathrm{NSs}$, exfoliated Ni-Fe-LDH NSs were also hybridized with $\mathrm{RuO}_{2}$ NSs to synthesize the NFR10 nanohybrid. Similar to the NCR nanohybrids, the NFR10 nanohybrid shows typical Bragg reflections of the Ni-FeLDH phase without any impurity peaks, indicating the presence of Ni-Fe-LDH in this material (see Figure S15 in the Supplementary Information). The formation of the homogeneously hybridized NFR10 material was confirmed by a TEM analysis (Fig. 6a), showing the anchoring of smaller LDH NSs with $(0,1,5)$ lattice fringes on the surfaces of larger $\mathrm{RuO}_{2} \mathrm{NSs}$ with $(1,0,-2)$ lattice fringes. The uniform hybridization between the $\mathrm{RuO}_{2}$ NSs and $\mathrm{Ni}-\mathrm{Fe}-\mathrm{LDH}$ NSs was further evidenced by the EFTEMelemental mapping results, which showed the $\mathrm{Ru}, \mathrm{Ni}, \mathrm{Fe}$, and $\mathrm{O}$ elements are homogeneously distributed in all parts of NFR10. As shown in the LSV curves in Fig. 6b, 

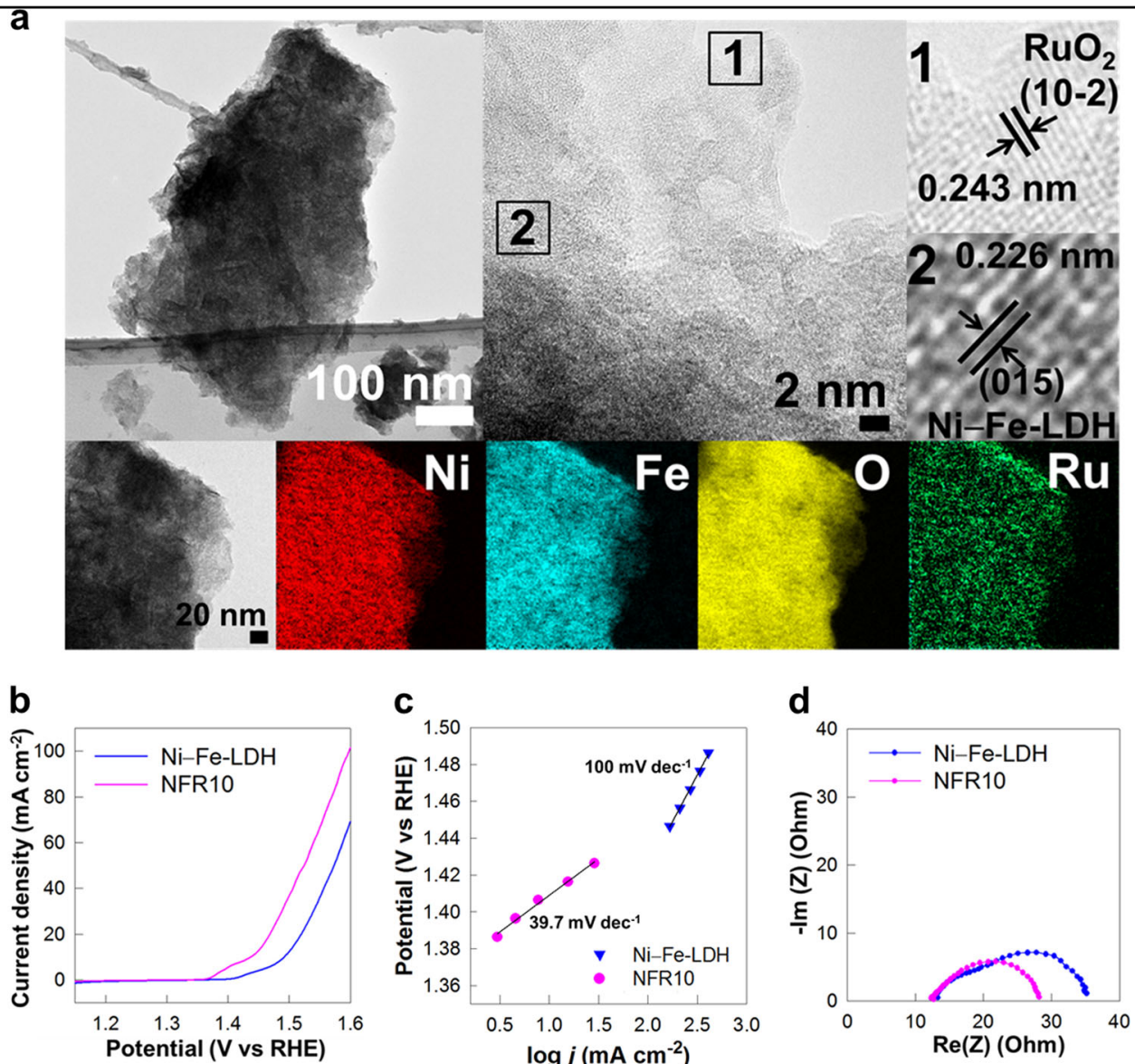

Fig. 6 Hybridization of cationic Ni-Fe-LDH with anionic $\mathrm{RuO}_{2}$ NS. a HR-TEM images and EFTEM-elemental maps. $\mathbf{b}$ LSV curves. c Tafel plot. and d EIS data for the NFR10 nanohybrid

NFR10 is much more electrocatalytically active for OER than the precursor Ni-Fe-LDH; NFR10 shows a very small overpotential of $207 \mathrm{mV}$ at $10 \mathrm{~mA} \mathrm{~cm}^{-2}$, which is much lower than that of pristine Ni-Fe-LDH $(261 \mathrm{mV})$. As plotted in Fig. 6c, NFR10 has a much smaller Tafel value of $39.7 \mathrm{mV} \mathrm{dec}^{-1}$ than that of Ni-Fe-LDH $(100 \mathrm{mV}$ $\mathrm{dec}^{-1}$ ), indicating an improvement in the OER kinetics upon hybridization with $\mathrm{RuO}_{2}$ NSs. This result highlights the beneficial effect of hybridization with $\mathrm{RuO}_{2} \mathrm{NSs}$ on the OER activity of $\mathrm{Ni}-\mathrm{Fe}-\mathrm{LDH}$. Table $\mathrm{S} 1$ in the Supplementary Information shows that the observed overpotential of NFR10 is one of the lowest values among those reported in previous studies on Ni-Fe-LDH-based electrocatalysts. According to the EIS analysis (Fig. 6d), the diameter of the semicircle reflecting the charge transfer resistance is smaller for NFR10 than pristine $\mathrm{Ni}-\mathrm{Fe}-\mathrm{LDH}$, confirming the promotion of charge transfer kinetics upon hybridization with $\mathrm{RuO}_{2} \mathrm{NSs}$. The present experimental findings provide strong evidence for the universal merit of $\mathrm{RuO}_{2} \mathrm{NSs}$ as an additive for exploring novel, efficient OER electrocatalysts in terms of strong electronic coupling with inorganic electrocatalysts and the resulting improvement in RDS kinetics.

\section{Discussion}

The combination theoretical and experimental study presented here clearly demonstrates that kinetic control of the RDS of an electrocatalytic reaction can provide a rational method to explore high-performance OER electrocatalysts with a remarkable decrease in overpotentials. The DFT calculations highlight the efficient role of $\mathrm{RuO}_{2}$ NSs as an electron acceptor in increasing the electrophilicity of hybridized inorganic layers and enhancing the surface adhesion of $\mathrm{OH}^{-}$and/or $\mathrm{OOH}^{*}$ species on the surface of $\mathrm{LDH}$. This leads to a remarkable decrease in the activation energy of the RDS upon hybridization with $\mathrm{RuO}_{2}$ NSs. Based on this theoretical calculation, highly efficient OER activities with remarkably lower overpotentials and excellent electrochemical stabilities can be achieved by intimate hybridization between electrocatalytically active LDH NSs and electron-withdrawing $\mathrm{RuO}_{2}$ NSs. Nanoscale hybridization between exfoliated 
$\mathrm{LDH}$ and $\mathrm{RuO}_{2}$ NSs can be easily achieved by an electrostatically derived self-assembly process because of their opposite surface charges. The resulting NCR10 and NFR10 boast excellent OER performances with very small overpotentials of 276 and $207 \mathrm{mV}$ at $10 \mathrm{~mA} \mathrm{~cm}^{-2}$, respectively, as well as improved charge transfer kinetics and electrochemical stability. It is worthwhile to note here that the DFT calculation predicts a much greater change in the threshold energies of Ni-Co-LDH upon $\mathrm{RuO}_{2}$ hybridization $(2.37 \mathrm{eV} \rightarrow 2.26 \mathrm{eV} ; 110 \mathrm{meV}$ decrease) compared with the shift of the on-set potential from $1.572 \mathrm{~V}$ for Ni-Co-LDH NS to $1.505 \mathrm{~V}$ for the NCR nanohybrid ( $68 \mathrm{mV}$ decrease). We determined that the theoretical comparison of a single $\mathrm{LDH}$ layer versus an $\mathrm{LDH}-\mathrm{RuO}_{2}$ bilayer (corresponding to the case when every LDH layer develops an interaction with $\mathrm{RuO}_{2}$ ) resulted in a more drastic change in the calculated overpotential than the experimental values. Even with the much smaller content of $\mathrm{RuO}_{2} \mathrm{NSs}(1 \mathrm{wt} \%$ ) in NCR10 compared with that in the theoretical model of a 1:1 LDH- $\mathrm{RuO}_{2}$ bilayer, NCR10 displays an OER activity slightly inferior but somewhat comparable to the theoretically predicted activity, highlighting the high efficiency of $\mathrm{RuO}_{2}$ hybridization in increasing the electrocatalytic activity of the LDH. The present electrochemical results clearly demonstrate the effectiveness of hybridization with electron-withdrawing inorganic NSs in remarkably improving the electrocatalytic functionality of polar, inorganic solids. Controlling the RDS via hybridization with a small amount of electronwithdrawing $\mathrm{RuO}_{2}$ NSs provides a novel, rational method to explore economically feasible efficient electrocatalysts. In comparison with previously reported, efficient, $\mathrm{RuO}_{2}$-based OER electrocatalysts showing low overpotentials of $360 \mathrm{mV}$ for commercial $20 \mathrm{wt} \% \mathrm{RuO}_{2} / \mathrm{C}$ (Ru: $\sim 14 \mathrm{wt} \%)^{39}, 290 \mathrm{mV}$ for $\mathrm{RuO}_{2}$-loaded on $\mathrm{Ti}$ plates (Ru: $\sim 74 \mathrm{wt} \%)^{40}$, and $220 \mathrm{mV}$ for (100)-oriented rutile $\mathrm{RuO}_{2}(\mathrm{Ru}: \sim 74 \mathrm{wt} \%)^{41}$, the present NFR10 nanohybrid with a much smaller $\mathrm{Ru}$ content of $\sim 0.7 \mathrm{wt} \%$ displays a much lower overpotential of $207 \mathrm{mV}$ at $10 \mathrm{~mA} \mathrm{~cm}^{-2}$, highlighting the usefulness of the present synthetic strategy in exploring novel, efficient electrocatalysts. The very low Ru content of the present nanohybrid provides this material with a high level of economic feasibility over that of conventional $\mathrm{RuO}_{2}$-based OER electrocatalysts. It is worthwhile to mention that despite the rarity of $\mathrm{Ru}$, the price of $\mathrm{Ru}$ is still 12 times cheaper than that of Ir, which is also utilized as an OER electrocatalyst $^{48}$, underscoring the validity of the present nanohybrid as an economically feasible electrocatalyst. Considering the fact that the 2D morphology of inorganic NSs provides effective anchoring sites for diverse inorganic electrocatalysts, the present synthetic strategy with electron-withdrawing or electron-donating inorganic NSs will evoke a great deal of research on the development of new, high-performance, hybrid electrocatalysts. A high flexibility in the compositional control of inorganic NSs via cation- and anion-substitution allows us to optimize the electronic structure and ability to accept or donate electron density of hybridized electrocatalysts ${ }^{49}$. Our current project is exploring novel, economically feasible, efficient electrocatalysts for HER, ORR, and $\mathrm{CO}_{2}$ reduction reaction via hybridization of nanostructured transition metal hydroxides/oxides with electron-withdrawing/-donating inorganic NSs.

\section{Acknowledgements \\ This work was supported by the National Research Foundation of Korea (NRF) grant funded by the Korea government (MSIP) (No. NRF- \\ 2017R1A2A1A17069463) and by the Korea government (MSIT) (No. NRF- 2017R1A5A1015365). The experiments at PAL were supported in part by MOST and POSTECH. \\ Author details \\ 'Center for Hybrid Interfacial Chemical Structure (CICS), Department of Chemistry and Nanoscience, College of Natural Sciences, Ewha Womans University, Seoul 03760, Republic of Korea. ${ }^{2}$ Department of Chemistry and Graduate School of EEWS, Korea Advanced Institute of Science and Technology (KAIST), Daejeon 34141, Republic of Korea. ${ }^{3}$ National Institute for Nanomaterials Technology (NINT), Pohang University of Science and Technology (POSTECH), Pohang 37673, Republic of Korea}

Conflict of interest

The authors declare that they have no conflict of interest.

\section{Publisher's note}

Springer Nature remains neutral with regard to jurisdictional claims in published maps and institutional affiliations.

Supplementary information is available for this paper at https://doi.org/ 10.1038/s41427-018-0060-3.

Received: 30 October 2017 Revised: 6 March 2018 Accepted: 25 May 2018. Published online: 23 July 2018

\section{References}

1. Su, D. et al. Ruthenium nanocrystal decorated vertical graphene nanosheets@Ni foam as highly efficient cathode catalysts for lithium-oxygen batteries. NPG Asia Mater. 8, e286 (2016).

2. Park, S., Shao, Y., Liu, J. \& Wang, Y. Oxygen electrocatalysts for water electrolyzers and reversible fuel cells: status and perspective. Energy Environ. Sci. 5, 9331-9344 (2012)

3. Diaz-Morales, O. et al. Iridium-based double perovskites for efficient water oxidation in acid media. Nat. Commun. 7, 12363 (2016).

4. Su, J. et al. Ruthenium-cobalt nanoalloys encapsulated in nitrogen-doped graphene as active electrocatalysts for producing hydrogen in alkaline media. Nat. Commun. 8, 14969 (2017)

5. Viswanathan, V., Hansen, H. A. \& Nørskov, J. K. Selective electrochemical generation of hydrogen peroxide from water oxidation. J. Phys. Chem. Lett. 8, 1157-1160 (2017).

6. Zhong, H.-x. et al. In situ anchoring of $\mathrm{CO}_{9} \mathrm{~S}_{8}$ nanoparticles on $\mathrm{N}$ and $\mathrm{S}$ codoped porous carbon nanotube as bifunctional oxygen electrocatalyst. NPG Asia Mater. 8, e308 (2016)

7. Zheng, Y. et al. Molecule-level $\mathrm{g}-\mathrm{C}_{3} \mathrm{~N}_{4}$ coordinated transition metals as a new class of electrocatalysts for oxygen electrode reaction. J. Am. Chem. Soc. 139, 3336-3339 (2017).

8. Song, F. \& Hu, X. Exfoliation of layered double hydroxides for enhanced oxygen evolution catalysis. Nat. Commun. 5, 4477 (2014) 
9. Friebel, D. et al. Identification of highly active $\mathrm{Fe}$ sites in $(\mathrm{Ni}, \mathrm{Fe}) \mathrm{OOH}$ for electrocatalytic water splitting. J. Am. Chem. Soc. 137, 1305-1313 (2015).

10. Lu, Z. et al. Ternary NifeMn layered double hydroxides as highly-efficient oxygen catalysts. Chem. Commun. 52, 908-911 (2016).

11. Hunter, B. M., Hieringer, W., Winkler, J. R., Gray, H. B. \& Muller, A. M. Effect of interlayer anions on [NiFe]-LDH nanosheet water oxidation activity. Energy Environ. Sci. 9, 1734-1743 (2016).

12. Jin, X., Lim, J., Lee, N.S. \& Hwang, S.J. A powerful role of exfoliated metal oxide $2 \mathrm{D}$ nanosheet as additives for improving electrocatalyst functionality of graphene. Electro. Acta 235, 720-729 (2017).

13. Tang, C., Wang, H. F., Zhu, X. L., Li, B. Q. \& Zhang, Q. Advances in hybrid electrocatalysts for oxygen evolution reactions: rational integration of NiFe layered double hydroxides and nanocarbon. Part. Part. Syst. Charact. 33 473-486 (2016).

14. Wu, J. et al. A highly active oxygen evolution electrocatalyst: ultrathin $\mathrm{CoNi}$ double hydroxide/CoO nanosheets synthesized via interface-directed assembly. Nano Res. 9, 713-725 (2016).

15. Rossmeisl, J., Logadottir, A. \& Nørskov, J. K. Electrolysis of water on (oxidized) metal surfaces. Chem. Phys. 319, 178-184 (2015).

16. Rossmeisl, J., Qu, Z.-W., Zhu, H., Kroses, G.-J. \& Nørskov, J. K. Electrolysis of water on oxides surfaces. J. Electroanal. Chem. 607, 83-89 (2007)

17. Lee, J. M. et al. A conductive hybridization matrix of $\mathrm{RuO}_{2}$ two-dimensional nanosheets: a hybrid-type photocatalyst, Angew. Chem. Int'l. Ed. 55, 8546-8550 (2016).

18. Lee, $\mathrm{S}$. et al. Superior additive of exfoliated $\mathrm{RuO}_{2}$ nanosheet for optimizing the electrode performance of metal oxide over graphene. J. Phys. Chem. C. 120, 11786-11796 (2016).

19. Kresse, G. \& Furthmüller, J. Efficiency of ab-initio total energy calculations for metals and semiconductors using a plane-wave basis set. Comput. Mat. Sci. $\mathbf{6}$, 15-50 (1996).

20. Kresse, G. \& Furthmüller, J. Efficient iterative schemes for $a b$ initio totalenergy calculations using a plane-wave basis set. Phys. Rev. B 54, 11169-11186 (1996).

21. Blöchl, P. E. Projector augmented-wave method. Phys. Rev. B 50, 17953-17979 (1994).

22. Kresse, G. \& Joubert, D. From ultrasoft pseudopotentials to the projector augmented-wave method. Phys. Rev. B 59, 1758-1775 (1999).

23. Perdew, J. P., Burke, K. \& Ernzerhof, M. Generalized gradient approximation made simple. Phys. Rev. Lett. 77, 3865-3868 (1996).

24. Dudarev, S. L., Botton, G. A., Savrasov, S. Y., Humphreys, C. J. \& Sutton, A. P. Electron-energy-loss spectra and the structural stability of nickel oxide: an LSDA+U study. Phys. Rev. B 57, 1505-1509 (1998).

25. Qian, L. et al. Trinary layered double hydroxides as high-performance bifunctional materials for oxygen electrocatalysis. Adv. Energy Mater. $\mathbf{5}$ 1500245 (2015).

26. Sanville, E., Kenny, S. D., Smith, R. \& Henkelman, G. An improved gridbased algorithm for Bader charge allocation. J. Comp. Chem. 28, 899-908 (2007).

27. Henkelman, G., Arnaldsson, A. \& Jönsson, H. A fast and robust algorithm for Bader decomposition of charge density. Comput. Mater. Sci. 36, 254-360 (2006).

28. Yu, M. \& Trinkle, D. R. Accurate and efficient algorithm for Bader charge integration. J. Chem. Phys. 134, 064111 (2011).

29. Momma, K. \& Izumi, F. VESTA 3 for three-dimensional visualization of crystal, volumetric and morphology data. J. Appl. Crystallogr. 44, 1272-1276 (2011).
30. Wang, V., Xiao, W., Ma, D.-M., Liu, R.J. \& Yang, C.-M. Structural, electronic, and optical properties of $\mathrm{GalnO}_{3}$ : A hybrid density functional study, J. Appl. Phys. 115, 043708 (2014)

31. Linstrom, P. J. \& Mallard, W. G. (eds.) NIST Chemistry WebBook, NIST standard reference Database number 69, National Institute of Standards and Technology, Gaithersburg MD, 20899, (retrieved April 27, 2017).

32. Bard, A. J. \& Faulkner, L. R. Electrochemical Methods. 2nd edn (John Wiley \& Sons, Inc: New York, 2001).

33. Kurth, S., Perdew, J. P. \& Blaha, P. Molecular and solid-state tests of density functional approximations: LSD, GGAs, and meta-GGAs. Int. J. Quantum Chem 75. 889-909 (1999).

34. Fukuda, K. et al. Synthesis of nanosheet crystallites of ruthenate with an a-NaFeO 2 -related structure and its electrochemical supercapacitor property. Inorg. Chem. 49, 4391-4393 (2010).

35. Jiang, J., Zhang, A., Li, L. \& Ai, L. Nickel-cobalt layered double hydroxide nanosheet as high performance electrocatalyst for oxygen evolution reaction. J. Power Sources 278, 445-451 (2015).

36. Coronado, E. et al. Spontaneous magnetization in Ni-Al and Ni-Fe layered double hydroxides. Inorg. Chem. 47, 9103-9110 (2008).

37. Dong, Y. et al. A first-principles study of oxygen formation over NiFe-layered double hydroxides surface. Catal. Lett. 145, 1541-1561 (2015).

38. García-Mota, M. et al. Importance of correlation in determining electrocatalytic oxygen evolution activity on cobalt oxides. J. Phys. Chem. C. 16, 21077-21082 (2010).

39. Li, Y., Hasin, P. \& Wu, Y. Ni $\mathrm{Ni}_{x} \mathrm{CO}_{3-x} \mathrm{O}_{4}$ nanowire arrays for electrocatalytic oxygen evolution. Adv. Mater. 22, 1926-1929 (2010).

40. Jiang, Z.-J. \& Jiang, Z. Interaction induced high catalytic activities of $\mathrm{CoO}$ nanoparticles grown on nitrogen-doped hollow graphene microspheres for oxygen reduction and evolution reactions. Sci. Rep. 6, 27081 (2016).

41. Pu, Z., Luo, Y., Asiri, A. M. \& Sun, X. Efficient electrochemical water splitting catalyzed by electrodeposited nickel diselenide nanoparticles based film ACS Appl. Mater. Inerfaces 8, (4718-4723 (2016).

42. Stoerzinger, K. A., Qiao, L., Biegalski, M. D. \& Shao-Horn, Y. Orientation dependent oxygen evolution activities of rutile $\mathrm{IrO}_{2}$ and $\mathrm{RuO}_{2}$. J. Phys. Chem. Lett. 5, 1636-1641 (2014).

43. Man, I. C. et al. Universality in oxygen evolution electrocatalysis on oxide surface. ChemCatChem. 3, 1159-1165 (2011).

44. Zhao, Y., Hernandez-Pagan, E. A., Vargas-Barbosa, N. M., Dysart, J. L. \& Mallouk, T. E. A high yield synthesis of ligand-free iridium oxide nanoparticles with high electrocatalytic activity. J. Phys. Chem. Lett. 2, 402-406 (2011).

45. Stoerzinger, K. A., Ziao, L., Biegalski, M. D. \& Shao-Horn, Y. Orientationdependent oxygen evolution activities of rutile $\mathrm{IrO}_{2}$ and $\mathrm{RuO}_{2}$. J. Phys. Chem. Lett. 5, 1636-1641 (2014).

46. Xia, W.-Y., Li, N., Li, Q.Y., Ye, K.H. \& Xu, C.-W. Au-NiCO $\mathrm{O}_{4}$ supported on threedimensional hierarchical porous graphene-like material for highly effective oxygen evolution reaction. Sci. Rep. 6, 23398 (2016).

47. Wang, Q. et al. Zinc-air batteries: NiFe layered double hydroxide nanoparticles on $\mathrm{Co}$, $\mathrm{N}$-codoped carbon nanoframes as efficient bifunctional catalysts for rechargeable zinc-air batteries. Adv. Mater. 7, 1700467 (2017).

48. InvestmentMine Home page. http://www.infomine.com/investment/metalprices/ (accessed Aug 5, 2016)

49. Lee, J. M. et al. A crucial of Rh substituent ion in photoinduced internal electron transfer and enhanced photocatalytic activity of CdS- $\mathrm{T}_{\left(5.2^{-} \times\right)} \mathrm{Rh}_{\times / 2} \mathrm{O}_{2}$ nanohybrids. Small 11, 5771-5780 (2015). 